\title{
Der Impact-Faktor (IF) - ein fehlerhaft eingesetzter Qualitäts-Parameter
}

\author{
Bodo Hertsch und Hans D. Lauk
}

\section{Ein Kommentar zu dem Artikel von W. Haße und R. J. Fischer}

Der Impact-Faktor (IF) oder genaver Journal Impact-Faktor (JIF) einer Fachzeitschrift soll messen, wie oft andere Zeitschriften einen Artikel aus ihr in Relation zur Gesamtzahl der dort veröffentlichten Artikel zitieren. Je höher der Impact-Faktor, desto angesehener ist eine Zeitschrift. Dies wirkt sich auf die akademische Beurteilung von Wissenschaftlern aus. Wer in Zeitschriften mit einem höheren Impact-Faktor publiziert, hat größere Karrierechancen. Die Berechnung des ImpactFaktors (IF) erfolgt innerhalb einer 3-Jahres-Spanne nach folgender Formel:

Zahl der Zitate im Bezugsjahr auf die Artikel der vergangenen 2 Jahre

Zahl der Artikel in den vergangenen 2 Jahren

Daraus folgt, dass es keinen JIF für das laufende Jahr geben kann (Zitat aus Wikipedia, der freien Enzyklopädie). Der Impact-Faktor wird v.a. in den Naturwissenschaften und der Medizin verwendet. Die Aussagekraft ist weltweit sehr umstritten, d.h. in der heutigen Zeit wird sie aufgrund eines umfangreichen Schrifttums völlig verneint. Das ergibt sich schon aus den Überschriften einzelner Artikel zum Thema Impact-Faktor, wie z.B. Sidney Bloch and Garry Walter (2001, Australian and New Zealand Journal of Psychiatry 35, 563-568) "The impact factor: time for change". Die Liste der Artikel mit ablehnender
Tendenz ist weltweit sehr groß. Als Gründe für die Ablehnung werden insbesondere in der Medizin angegeben:

1. Der Anspruch als Qualitätsparamter für wissenschaftliche Publikationen sowie für universitäre und außer-universitäre Leistungen in der Wissenschaft und Forschung ist aufgrund der quantitativen Ausrichtung dieses Bewertungsfaktors nicht geeignet für eine qualitative Beurteilung. Dieses System ist insofern fehlerhaft, weil alle Referenzen, gleich ob sie Artikel, Editorials, Meetings, Letters oder Conference Proceedings betreffen, als Zitate berücksichtigt werden. Diese werden im Zähler als Zitierung gezählt, nicht aber als Artikel im Nenner. Folglich ergibt sich daraus ein besonders hoher Impact-Faktor. Das hat zur Folge, dass auch besonders viele Selbstzitierungen eingesetzt werden. Auch die Zeitspanne von nur 2 Jahren berücksichtigt nicht, dass viele Themenfelder, und das betrifft insbesondere die Veterinärmedizin, eine erheblich längere Zeit brauchen, um in der Wissenschaft durch nevere Artikel wieder aufgearbeitet zu werden. Man ist sich weltweit jetzł darüber einig, dass der IF ein einfaches verfügbares bibliometrisches Hilfsmittel ist aber mit sehr limitierter Aussagekraft über die Qualität einer Zeitschrift.

2. Ein weiteres Problem, das insbesondere die veterinärmedizinischen Zeitschriften betrifft, liegt in der Landessprache, in der die Artikel publiziert werden. Die Autoren sind gezwun-

Tab. 1 Impact-Faktoren ausgewählter englisch- und deutschsprachiger Titel

\begin{tabular}{lc}
\hline Titel & Impact-Faktor \\
\hline Veterinary Research & 3,579 \\
Preventive Veterinary Medicine & 2,121 \\
Equine Veterinary Journal (publiziert ausschließlich pferdemedizinische Beiträge) & 1,837 \\
Journal of American Veterinary Medicine & 1,714 \\
Veterinary Record & 1,504 \\
\hline & 0,976 \\
\hline Berliner Münchner Tierärztliche Wochenschrift & 0,614 \\
Schweizer Archiv für Tierheilkunde & 0,523 \\
Deutsche Tierärztliche Wochenschrift & 0,320 \\
Pferdeheilkunde (publiziert ausschließlich pferdemedizinische Beiträge) & 0,256 \\
Tierärztliche Praxis & 0,239 \\
Praktischer Tierarzł & 0,225 \\
Wiener Tierärztliche Monatsschrift & 0,179 \\
Vlaams Diergeneskunde & 0,167 \\
Tierärztliche Umschau & \\
\hline
\end{tabular}


gen, auch englisch-sprachige Artikel zu veröffentlichen oder überhaupt einen völligen Übergang zu dieser Sprache zu vollziehen, um überhaupt zitiert zu werden. Das führt auch dazu, dass z.B. deutsch-sprachige veterinärmedizinische Zeitschriften einen Übergang zur englischen Sprache vollziehen, indem sie den Titel wechseln, wie z.B. das ehemalige Zentralblatt für Veterinärmedizin, das nunmehr als Journal of Veterinary Medicine erscheint.

3. Für Autoren aus dem nicht-englisch-sprachigen Raum ist es eine große, nahezu unlösbare Aufgabe, in einer englischsprachigen Zeitschrift der Veterinärmedizin Artikel anzubieten. Zahlreiche deutsche Autoren, um nicht zu sagen alle, die aus dem deutsch-sprachigen Raum in englischer Sprache verfasste Artikel an englische oder amerikanische Zeitschriften einreichen, werden permanent wegen mangelnder Qualität der englischen Sprache abgelehnt und dies, obwohl die Übersetzungen meist nicht von den Autoren selbst, sondern von amerikanischen oder englischen Muttersprachlern erfolgt sind. Insofern stellt sich die Frage, ob der Ablehnungsgrund "poor english" nicht einen "Schein"-Grund darstellt. Über mögliche Hintergründe lässt sich nur spekuliere. Sollten nicht vielmehr Zeitschriften- sowie Sprachen-unabhängige, neutrale Wissenschaftler bzw. auch Bibliothekare übergreifend und zentral formiert ein Beurteilungsgremium bilden.

Vergleicht man die veterinärmedizinischen Zeitschriften, aber insbesondere die Zeitschriften, die sich auch mit Themen aus der Pferdeheilkunde beschäftigen, ist die Differenz zwischen deutsch- und englisch-sprachigen Zeitschriften enorm, obwohl von der fachlichen Qualität diese Unterschiede nicht zu bestätigenund in keiner Weise zu rechtfertigen sind. Tabelle 1 zeigt exemplarisch die IF aus dem Jahr 2010 für englisch-sprachige Zeitschriften.

Wie im Alltag sind wir auch in der Wissenschaft in einem Umbruch begriffen. Der Wissenschafts- "Markt" findet in englischer Sprache statt. Die deutschen Ärzte, einschließlich der Tierärzte, sind bzgl. ihres beruflichen Wissens "Zweitverwerter". Nur wenige Ärzte lesen englisch-sprachige Zeitschriften. Die Mehrheit verlässt sich auf referierte Artikel und Aufbereitungen in deutscher Sprache (Fendrich und Rothmund 2010). Dem bestehenden Wunsch, uns diesem englisch-sprachigen System anzupassen, werden zurzeit jedoch aufgrund der Beurteilung durch die Ausschüsse der ausländischen Journale Grenzen gesetzt. In angelsächsischen Arbeiten findet das anderssprachige Schrifttum in der Regel keine Berücksichtigung, obwohl nahezu für jede Fachzeitschrift eine englische Zusammenfassung und englische Schlagwörter zu den Artikeln verfasst werden müssen. Es entsteht der Eindruck, dass dies lediglich der Speisung bibliothekarischer Suchmaschinen dient und nicht der inhaltlichen Weitergabe von wissenschaftlich erarbeiteten Informationen. Das trifft nicht nur die Pferdemedizin, sondern allgemein die Veterinärmedizin und auch in vollem Umfang die Humanmedizin - ein unmöglicher Zustand der weltweit diskutiert werden muss.

Der Impact-Faktor als Beurteilungsmaß oder "Gütesiegel" für eine Fachzeitschrift erfüllt, wenn überhaupt, für die wissenschaftliche Literatur in den USA/UK bzw. den englisch-sprachigen Ländern und Periodika seinen Zweck. Für alle anderen Länder, insbesondere für Deutschland, Österreich und die Schweiz, wirkt er sich in der bisherigen Praxis der weitgehend kritiklosen Anerkennung durch die hiesigen medizinischen und tiermedizinischen Wissenschaften diskriminierend und destruierend aus. Es stellt sich immer wieder erneut die Frage, warum man sich diesem Diktat freiwillig unterwirft.

Ein Beispiel für die verzerrende Auswirkung des IF auf insbesondere die kleineren wissenschaftlichen Fachzeitschriften ist die "Pferdeheilkunde". Nach 27 Jahren wird diese Zeitschrift von PubMed der U. S. National Library of Medicine (NLM), nach wie vor nicht zur Kenntnis genommen mit der Folge, dass unsere Veröffentlichungen im englisch-sprachigen Raum nur unzureichend bekannt und zitiert werden. Der aus diesem Grunde im Vergleich zu den englisch-sprachigen Fachzeitschriften niedrigere IF für die "Pferdeheilkunde" verhindert widerum deren Aufnahme in die US-Zitationsdatenbank. Aus diesem Circulus vitiosus wäre möglicherweise nur zu entkommen, wenn die Zeitschrift gänzlich auf die deutsche Sprache verzichtete. Aber auch nur möglicherweise, denn andere Zeitschriften, die Jahre lang in PubMed vertreten waren - auch rein englisch-sprachige Titel -, erscheinen dort plötzlich nicht mehr, weil sie, unabhängig von der Zitationsquote, irgendwelchen Anforderungskriterien nicht mehr genügen. Wieder andere Zeitschriften, durchaus auch solche in nicht englischer Sprache, erscheinen in PubMed, ohne dass dies nachzuvollziehen wäre. Offensichtlich wendet man die eigenen Regeln sehr willkürlich an. Die restriktive NLM-Politik des Ausschlusses nicht englischsprachiger Zeitschriften soll insbesondere in unserem Fachgebiet auch schon einmal dazu führen, dass in den USA Erkenntnisse und Methoden neu ge- bzw. erfunden werden, die bei uns schon bekannt und publiziert sind. Erschwerend kommt hinzu, dass die Mehrheit der deutsch-sprachigen Autoren für ihre Literaturrecherche überwiegend nur PubMed nutzt, mit der Konsequenz, dass die dort nicht vertretenen deutsch-sprachigen Zeitschriften nicht einmal mehr von den "eigenen" Leuten zitiert werden. Welch unsinniger und unwissenschaftlicher Zustand!

Mit der Einrichtung des "Pferdeheilkunde"-Fundus wurde versucht, einerseits die deutsch-sprachigen Tierärzte dazu anzuhalten, die Arbeiten aus dieser Zeitschrift zu zitieren, und andererseits den englisch-sprachigen Kollegen Einsicht in die dort publizierten wissenschaftlichen Arbeiten zu ermöglichen. Leider gelingt das nur unzureichend, weil sich wenige Autoren die Mühe machen, bei der Recherche für ihre Veröffentlichungen mehr als eine Zitationsdatenbank, wie z.B. PubMed, abzufragen mit der Konsequenz, dass die deutsch-sprachigen Publikationen selbst in deutsch-sprachigen Zeitschriften immer weniger und die englischen Publikationen immer häufiger zitiert werden. Ein fataler Verlust der Kenntnis wichtiger wissenschaftlicher Arbeit.

Dem IF-Diktat ist nur zu entkommen, wenn sich, wie Hasse und Fischer beschreiben, ein alternatives europäisches Bewertungssystem für wissenschaftliche Arbeiten etabliert, das vor allem die Bedingungen der Mehrsprachigkeit respektiert und alle wissenschaftlichen Zeitschriften berücksichtigt, welche die internationalen Anforderungen an hochwertige Fachperiodika erfüllen. Ein Maß für das Zitierverhalten wissenschaftlicher Autoren, als das der IF angesehnen werden muss, kann nicht gleichzeitig ein Qualitätsmerkmal für Fachzeitschriften darstellen.

Prof. Bodo Hertsch

Feldweg 2,14558 Tremsdorf

hertsch@eichenhof-tremsdorf.de 